\title{
The Master Catalogue of stars towards the Magellanic Clouds ${ }^{\star}$
}

\section{Multispectral surveys of the Large Magellanic Cloud}

\author{
N. Delmotte ${ }^{1,4}$, C. Loup ${ }^{2}$, D. Egret ${ }^{1}$, M.-R. Cioni ${ }^{3,4}$, and F. Pierfederici ${ }^{4}$ \\ 1 CDS, Observatoire Astronomique de Strasbourg, UMR 7550, Université Louis Pasteur, 67000 Strasbourg, France \\ e-mail: delmotte@astro.u-strasbg.fr; egret@astro.u-strasbg.fr \\ 2 Institut d'Astrophysique de Paris, CNRS UPR 341, 98bis Bld Arago, 75014 Paris, France \\ e-mail: loup@iap.fr \\ ${ }^{3}$ Leiden Observatory, University of Leiden, PO Box 9513, 2300 RA Leiden, The Netherlands \\ ${ }^{4}$ European Southern Observatory, ESO, K.-Schwarzschild-Str.-2, 85748 Garching, Germany \\ e-mail: mcioni@eso.org
}

Received 28 March 2002 / Accepted 5 September 2002

\begin{abstract}
The Master Catalogue of stars towards the Magellanic Clouds (MC2) ${ }^{\star \star}$ is a multi-wavelength reference catalogue. The current paper presents the first results of the MC2 project. We started with a massive cross-identification of the two recently released near-infrared surveys: the DENIS Catalogue towards the Magellanic Clouds (DCMC) with more than 1.3 million sources identified in at least two of the three DENIS filters $\left(I J K_{\mathrm{s}}\right)$ and the 2nd Incremental Release of the 2MASS point source catalogue $\left(J H K_{\mathrm{s}}\right)$ covering the same region of the sky. Both point source catalogues provide an unprecedented wealth of data on the stellar populations of the Magellanic Clouds (MCs). The cross-matching procedure has been extended to optical wavelength ranges, including the UCAC1 (USNO) and GSC2.2 catalogues. New cross-matching procedures for very large catalogues have been developed and important results on the astrometric and photometric accuracy of the cross-identified catalogues were derived. The cross-matching of large surveys is an essential tool to improve our understanding of their specific contents. This study has been partly supported by the ASTROVIRTEL ${ }^{\star \star \star}$ project that aims at improving access to astronomical archives as virtual telescopes.
\end{abstract}

Key words. galaxies: Magellanic Clouds - galaxies: stellar content - methods: statistical - methods: data analysis - catalogs astronomical data bases: miscellaneous

\section{Introduction}

The Magellanic Clouds (MCs) are among the best suitable places to study the stellar evolution outside the Milky Way, because of their proximity and their various stellar populations. Near-infrared surveys provide useful data for this kind of study because of their insensitivity to interstellar reddening. The Magellanic Clouds have been recently fully observed by two major infrared surveys: the DEep Near-Infrared Survey of the Southern Sky - DENIS (Epchtein et al. 1997) and the Two Micron All Sky Survey - 2MASS (Skrutskie et al. 1997). A Near-Infrared Point Source Catalogue

Send offprint requests to: N. Delmotte,

e-mail: delmotte@astro.u-strasbg.fr

* This work has been supported by ASTROVIRTEL, a project funded by the European Commission under FP5 Contract No. HPRI-CT-199900081 .

$\star \star$ http://vizier.u-strasbg.fr/MC2/

$\star \star \star$ http://www.stecf.org/astrovirtel/ towards the Magellanic Clouds, based on DENIS data, has been published (Cioni et al. 2000a; DCMC). The part of this catalogue devoted to the Large Magellanic Cloud (LMC) covers an area of $19.87 \times 16$ square degrees centered on $\left(5^{\mathrm{h}} 27^{\mathrm{m}} 20^{\mathrm{s}},-69^{\circ} 00^{\prime} 00^{\prime \prime}\right)$. To compile this catalogue, the objects were required to be detected in at least two of the three DENIS bands $I$ (Gunn-i, $0.79 \mu \mathrm{m}), J(1.22 \mu \mathrm{m}), K_{\mathrm{s}}(2.15 \mu \mathrm{m})$. The 2MASS project observed the whole Magellanic Clouds in three photometric bands: $J(1.23 \mu \mathrm{m}), H(1.63 \mu \mathrm{m})$ and $K_{\mathrm{s}}(2.15 \mu \mathrm{m})$. For this work we used only the data available from the 2nd Incremental Release PSC ${ }^{1}$, which do not cover two rectangular regions crossing the bar of the Large Magellanic Cloud and some cross-like gaps around bright stars $\left(4^{\mathrm{h}} 00^{\mathrm{m}} 00^{\mathrm{s}}<\mathrm{RA}<\right.$ $\left.7^{\mathrm{h}} 00^{\mathrm{m}} 00^{\mathrm{s}} ;-78^{\circ} 01^{\prime} 37^{\prime \prime}<\operatorname{Dec}<-60^{\circ} 48^{\prime} 00^{\prime \prime}\right)$.

The number of sources from both surveys are recorded in Table 1. Because of different sensitivity limits, DENIS sources detected only in the $I$ and $J$ bands are often detected in $H$ and

\footnotetext{
${ }^{1}$ http://www.ipac.caltech.edu/2mass/
} 

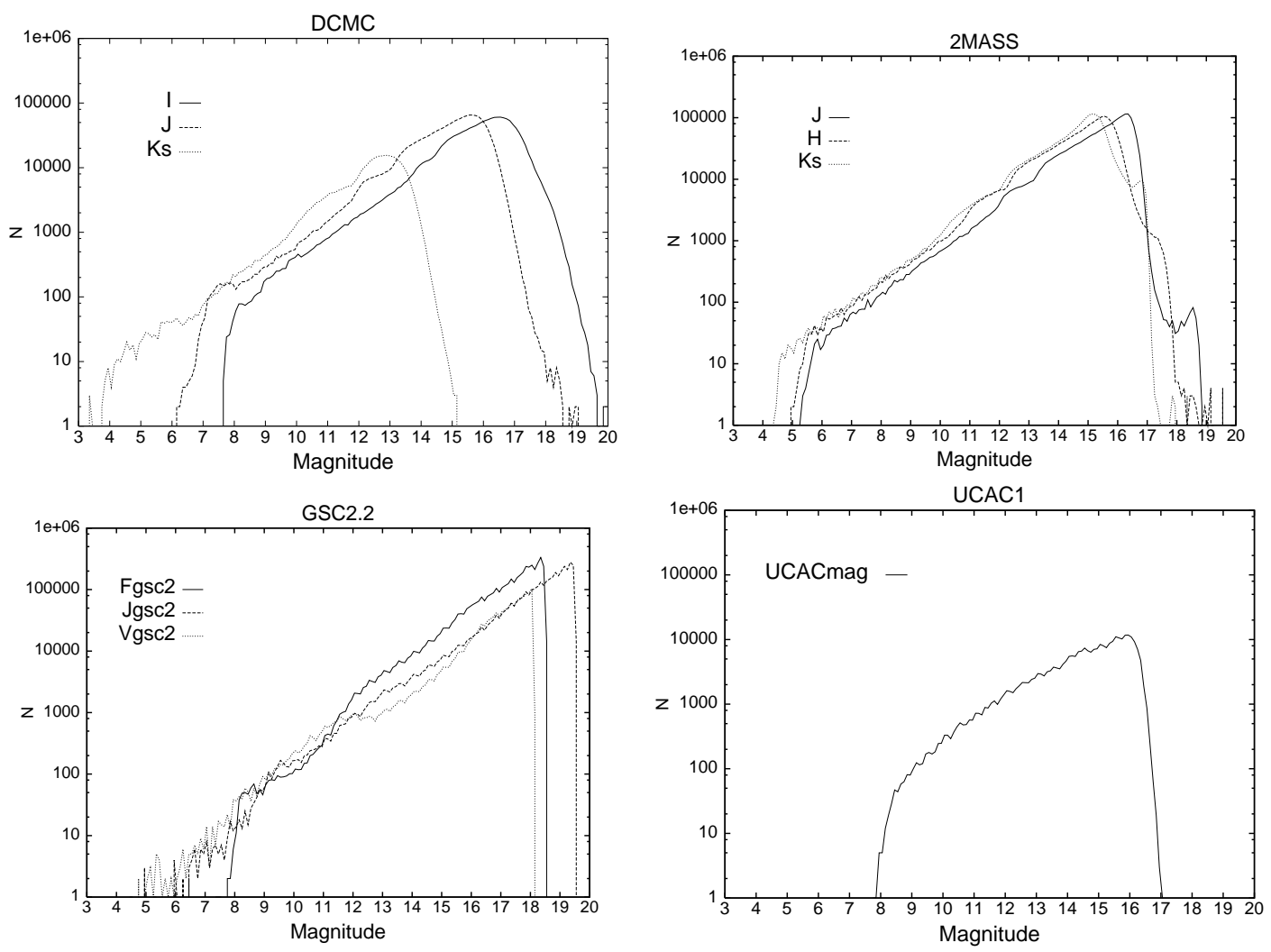

Fig. 1. Completeness diagrams for four major surveys covering the LMC. Each plot gives the number of sources per magnitude bin. The bin size is 0.1 mag. Note that: 2MASS observations (upper right) are deeper than DENIS observations (upper left) in the $K_{\mathrm{s}}$ band; the GSC2.2 magnitudes (bottom left) show a sharp cut-off; the UCAC1 magnitudes (bottom right) are only indicative, since the UCAC1 is not a photometric catalogue and furthermore this is only a preliminary catalogue, which means some improvements are expected in future releases.

$K_{\mathrm{s}}$ by 2MASS. 2MASS observations are more than one magnitude deeper than DENIS in the $K_{\mathrm{S}}$ channel (due to a better thermalization), while they are roughly equivalent in the $J$ channel (Fig. 1). Thus it appeared very interesting to cross-match the two catalogues to complete the spectral range of the DCMC $I J$-sources with the $H$ and $K_{\mathrm{s}}$ bands coming from 2MASS, though observations are not simultaneous.

More generally, cross-matching catalogues is highly relevant for completing the spectral or spatial coverage when there are missing or unpublished data. It is also a powerful tool to cross-validate the catalogues and search for discrepancies.

Cross-matching infrared (IR) with optical catalogues, such as DCMC/2MASS with the Guide Star Catalog II (GSC2.2), helps on producing new colour-magnitude and colour-colour diagrams, thus offering multispectral views of the LMC. In the cross-matching procedure we also included the proper motions from the USNO CCD Astrograph Catalogue (UCAC1), in order to discriminate MC members from foreground stars. The resulting MC2 catalogue provides an unprecedented basis for the study of stellar populations in the Magellanic Clouds and for further cross-identifications with catalogues at other wavelengths.

Section 2 gives an overview of each survey towards the LMC. Section 3 deals with the strategy developed to crossmatch the infrared DENIS and 2MASS catalogues. Following in Sect. 4 is a comparison of the DENIS and 2MASS photometric systems. In Sect. 5 we add the optical GSC2.2 and
Table 1. Number of sources as a function of detected wavebands in the DCMC and 2MASS catalogues.

\begin{tabular}{rr|rr}
\hline \hline \multicolumn{3}{c}{ LMC } \\
\hline \multicolumn{2}{c|}{ DCMC } & \multicolumn{2}{c}{$2 \mathrm{MASS}$} \\
\hline$I J K_{\mathrm{s}}$ & 297031 & $J H K_{\mathrm{s}}$ & 1996382 \\
$I J$ & 1151789 & $J K_{\mathrm{s}}$ & 66 \\
$I K_{\mathrm{s}}$ & 8724 & $J H$ & - \\
$J K_{\mathrm{s}}$ & 1897 & $H K_{\mathrm{s}}$ & 4 \\
& & $J$ & 11 \\
& & $H$ & - \\
& & $K_{\mathrm{s}}$ & 23 \\
& & Saturated & 259 \\
\hline Total & 1459441 & Total & 1996745 \\
\hline
\end{tabular}

UCAC1 catalogue to the cross-matching procedure. In Sect. 6 we present a few multispectral views of the stellar populations of the Clouds, based on the MC2 data.

\section{Data overview}

The present work is based on public data from 2MASS, as described in Sect. 1, and on DCMC data obtained from a local copy of the catalogue that includes the missing strips of the first release (the second release is currently under process). The GSC2.2 and the UCAC1 catalogues are now also publicly available. For each catalogue, a density map is produced (see 

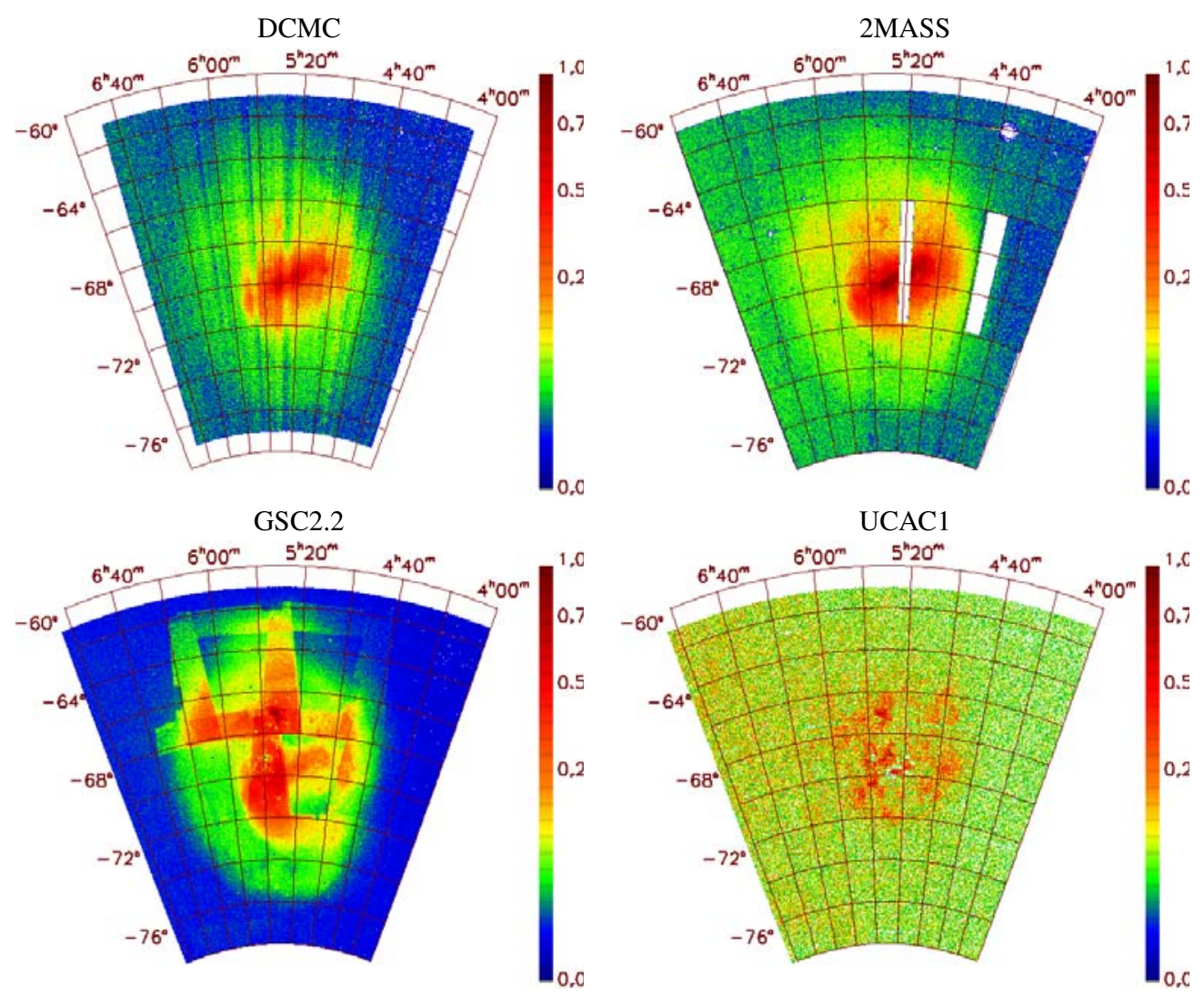

Fig. 2. Density maps of the LMC: DCMC (upper left), 2MASS (upper right), GSC2.2 (bottom left) and UCAC1 (bottom right) sources. The pixel size is $\sim 2.8^{\prime} \times 2.7^{\prime}$. Each map has been normalized to unity, but note that the highest pixel value reaches 208 for the DCMC, 186 for 2MASS, 656 for the GSC2.2 and 18 for the UCAC1.

Fig. 2). The higher concentration of sources in the upper left part of each plot is in the direction of the Galactic center.

Inhomogeneities in the magnitude limit of some DENIS strips are visible on the DCMC map. White areas in the 2MASS map denote missing data due to observations not yet released. The circular gap on the upper right corner corresponds to the semi-regular pulsating star HD 29712, which is also the second brightest star in the sky in $K_{\mathrm{s}}$ (after Betelgeuse) with a magnitude lower than -4 .

The GSC2. $2^{2}$ catalogue provides, in the direction of the LMC, $\quad\left(3^{\mathrm{h}} 59^{\mathrm{m}} 30^{\mathrm{s}}<\mathrm{RA}<7^{\mathrm{h}} 04^{\mathrm{m}} 30^{\mathrm{s}} ;-78^{\circ} 00^{\prime} 00^{\prime \prime}<\right.$ Dec $<$ $\left.-61^{\circ} 00^{\prime} 00^{\prime \prime}\right)$, the $F_{\mathrm{gsc} 2}, J_{\mathrm{gsc} 2}$, and $V_{\mathrm{gsc} 2}$ photographic bands for 6032541 entries. The $V_{\mathrm{gsc} 2}$ magnitudes are from short exposure $V$ plates used to deal with dense regions of the sky. The photographic magnitudes given in the GSC2.2 are in the natural systems of the photographic plates (emulsion/filter: IIIaF+OG590, IIIaJ+GG395, IIaD+W12). The photometric calibration is based on a Chebyshev polynomial fit to sequence stars from the Second Guide Star Photometric Catalogue (Bucciarelli et al. 2001) and the Tycho Catalog for the bright end. However the three magnitudes are not always present together. The $J_{\mathrm{gsc} 2}$ band is missing for the innermost parts of the LMC, but is replaced by the $V_{\mathrm{gsc} 2}$ band. Thus, when using the $V_{\mathrm{gsc} 2}$ band in the following colour-magnitude or colour-colour diagrams of this paper, one should keep in mind

\footnotetext{
${ }^{2}$ http://www-gsss.stsci.edu/gsc/gsc2/GSC2home.htm
}

that we are dealing with the central parts of the LMC only. The unusual patterns on the GSC2.2 density map match the HTM (Hierarchical Triangular Mesh) partitioning of the data, which is a recursive spatial indexing scheme dividing the unit sphere into spherical triangles. This is probably due to difficulties in producing the final catalogue for the most crowded regions.

The UCAC1 catalogue ${ }^{3}$ (Zacharias et al. 2000) contains, in the direction of the LMC, 267,103 entries $\left(3^{\mathrm{h}} 59^{\mathrm{m}} 30^{\mathrm{s}}<\mathrm{RA}\right.$ $\left.<7^{\mathrm{h}} 04^{\mathrm{m}} 28^{\mathrm{s}} ;-78^{\circ} 00^{\prime} 00^{\prime \prime}<\operatorname{Dec}<-61^{\circ} 00^{\prime} 00^{\prime \prime}\right)$. This is the preliminary version of an astrometric catalogue, which aims at increasing the number of optical sources with high positional accuracy. Proper motions are available, combining the UCAC1 with the USNO-A2.0 (Monet et al. 1998) positions for faint stars, and with older catalogues for bright stars. One magnitude, intermediate between Johnson $V$ and $R$, is provided (579-642 nm). White regions on the UCAC1 density map denote missing digitalization frames in the center of the bar, due to difficulties in extracting sources in overcrowded regions of the sky.

The catalogues used to build the MC2 present the following differences: the observational strategy that influences the homogeneity of the final data, the passbands, the characterized stellar populations and the number of sources. These factors have a strong impact on the results of our cross-matching.

\footnotetext{
${ }^{3}$ http://ad.usno.navy.mil/ad/ucac/
} 


\section{First step: Cross-matching DENIS and 2MASS}

\subsection{Cross-matching strategy}

Before running the cross-matching programs, we organized the original data, splitting most of the catalogues into smaller pieces. The DENIS observational strategy had been to divide the sky in strips of $30^{\circ}$ in Declination (Dec) and $12^{\prime}$ in Right Ascension (RA). To define subsamples, we split the DCMC catalogue by strip number because:

- our cross-matching algorithm is well adapted to data files with small extension in RA;

- the cross-matching criterion depends on the strip number as explained below.

There are 119 strip-files covering the LMC. For each strip-file, we extracted from the 2MASS catalogue all the point sources belonging to the same region of the sky. Matches between both catalogues are found by specifying a position search box of a few arcseconds, and comparing the coordinates of entries in both catalogues. The cross-matching program is executed for each strip, starting, each time, with two input files, one from DCMC and one from 2MASS. Both files have been previously sorted by declination, in order to optimize the crosscomparison procedures. Details about the procedure are as follows: for each record of the first file (say, DCMC) we search for all possible cross-matches in the second file (here 2MASS). Among the possible cross-matches, we only keep the one with the smallest difference in position as the most probable counterpart. The actual limits imposed to the positional difference $\Delta \alpha$ and $\Delta \delta$ depend upon the relative astrometry of the strip (see below).

\subsubsection{Finding discrepancies in the original catalogues}

Cross-matching by position works very well in most cases because the astrometry of DCMC and 2MASS is accurate enough (better than one arcsecond). 2MASS positions were reconstructed from the ACT reference catalogue (Urban et al. 1998), using the Tycho astrometry. The astrometric reference for DCMC positions is the USNO-A2.0 catalogue (Monet et al. 1998). The astrometric solution is global for a strip, minimizing possible inaccuracies of the USNO-A2.0 catalogue in the most crowded regions.

Consequently the match distance is smaller than $0.5^{\prime \prime}$ for the great majority of the stars. There is in principle no risk of confusion at such a small scale. While this is true in general, in practice the cross-matching exercise has proven to be a powerful tool to detect subsets of data which deviate from the perfect situation, and primarily areas suffering from problems in the astrometric or photometric calibration.

In some cases, field distortions in the DCMC affect the quality of the astrometry. To detect and quantify them, we proceeded strip by strip. We kept only well confirmed DCMC sources: $10.5 \leq I \leq 16.5$ and flags in the $I$ band equal to zero. We ran a cross-matching program based only on distances, with a searching box that goes up to $30^{\prime \prime}$. Between all the possible associations found, we kept only the association with $\left|J_{\text {DCMC }}-J_{2 \text { MASS }}\right| \leq 0.5$. The selection is done on magnitude because in case of field distortions, small distances are not reliable enough a criterion.

The relative shifts in RA and Dec are a function of the pixel coordinates of the camera. We found 11 strips affected by field distortions at a level larger than $2^{\prime \prime}$. We also searched for systematic shifts $\delta J$ and $\delta K_{\mathrm{s}}$ between DCMC and 2MASS magnitudes. Mean shifts have been computed for each strip. The diagrams corresponding to the positional and magnitude shifts are all available, strip by strip, on the MC2 web site ${ }^{4}$.

Such astrometric and magnitude shifts depend on the particular strip and had to be taken into account in the DENIS versus 2MASS cross-matching. Strategies for coping with them have been implemented, to allow a proper strip by strip crossmatching of both catalogues. We took advantage of the $J$ and $K_{\mathrm{S}}$ common magnitudes of the two surveys. A potential crossmatched source is thus validated not only on a positional criterion, but also on magnitude criteria.

\subsubsection{Defining a positional searching box}

Shifts in RA $(\delta \alpha)$ and Dec $(\delta \delta)$ being mainly a function of the pixel coordinates, they do vary inside one image, but are nearly the same for all the images of the strip. So it is better to use the statistics of the whole strip instead of one single image. Thus we can define a specific position search box for the strip. The size of the box will take into account the shifts in RA and Dec found for this strip number. The default size of the searching box when there are no shifts is $3^{\prime \prime}$. So we have now an enlarged and asymmetric searching box:

$\frac{\delta \alpha_{\min }^{\prime \prime}-3^{\prime \prime}}{\cos \delta}<\alpha_{\mathrm{DCMC}}-\alpha_{2 \mathrm{MASS}}<\frac{\delta \alpha_{\max }^{\prime \prime}+3^{\prime \prime}}{\cos \delta}$

$\delta \delta_{\text {min }}^{\prime \prime}-3^{\prime \prime}<\delta_{\text {DCMC }}-\delta_{2 \text { MASS }}<\delta \delta_{\text {max }}^{\prime \prime}+3^{\prime \prime}$,

where $\delta \alpha_{\min }, \delta \alpha_{\max }, \delta \delta_{\min }, \delta \delta_{\max }$ are the minimum and maximum shifts in RA and Dec (arcseconds). Note that the searching box has a complex shape, since $\delta \alpha_{\text {min }} \neq \delta \alpha_{\text {max }}$ and $\delta \delta_{\text {min }} \neq$ $\delta \delta_{\text {max }}$. This box is used to optimise the probability to find the correct cross-matching, even in distorted images.

\subsubsection{Selection on magnitudes}

Between all the possible associations found in Sect. 3.1.2, we must keep the best one. We have seen that keeping the association with the smallest distance is no more a reliable criterion because of field distortions. So we have to check the compatibility in magnitude for each association, after applying on the strip data the associated mean magnitude shifts $\langle\delta J\rangle$ and $<\delta K_{\mathrm{s}}>$ computed in Sect. 3.1.1 above.

- If $K_{\mathrm{s}}$ is not detected in the catalogues, the selection is done on $J$. The following relation has to be true to keep the association:

$$
|\delta J-<\delta J>| \leq w \times \sqrt{\sigma_{J_{\mathrm{DCMC}}}^{2}+\sigma_{J_{2 \mathrm{MASS}}}^{2}},
$$

\footnotetext{
${ }^{4}$ http://vizier.u-strasbg.fr/MC2/
} 
where $w=2$ is a weight, and $\sigma_{J_{\mathrm{DCMC}}}$ and $\sigma_{J_{2 \mathrm{MASS}}}$ are the relative photometric uncertainties as quoted in both catalogues. Relative uncertainties are in general very small for bright stars, less than 0.01 mag. However, uncertainties on the absolute calibration are much larger: about 0.1 mag for the DCMC. If we apply abruptly the above criterion, we will lose many cross-identifications for the stars with small relative uncertainties. We thus need to refine the selection criterion and consider two cases:

$$
\begin{aligned}
& \text { if } \quad w \times \sqrt{\sigma_{J_{\mathrm{DCMC}}}^{2}+\sigma_{J_{2 \mathrm{MASS}}}^{2}} \leq \Delta J \text { then } \\
& |\delta J-<\delta J>| \leq \Delta J \\
& \text { else if } \quad w \times \sqrt{\sigma_{J_{\mathrm{DCMC}}}^{2}+\sigma_{J_{2 \mathrm{MASS}}}^{2}}>\Delta J \\
& \text { then } \quad|\delta J-<\delta J>| \leq w \times \sqrt{\sigma_{J_{\mathrm{DCMC}}}^{2}+\sigma_{J_{2 \mathrm{MASS}}}^{2}}
\end{aligned}
$$

where $\Delta J=0.45$ is the estimated full-width at halfmaximum of the $\delta J$ distribution;

- if $J$ is not detected in the catalogues, the selection is done on $K_{\mathrm{s}}$ as above but this time we have $\Delta K_{\mathrm{s}}=0.60$;

- if $J$ and $K_{\mathrm{s}}$ are detected in both catalogues, the selection is done on $J$ and then on $J-K_{\mathrm{s}}$;

- if neither $J$ nor $K_{\mathrm{s}}$ are detected in the catalogues, the association is lost.

Applying these criteria, if there are still more than one possible association for one DCMC source, then we keep the association with the smallest $\delta J$ or $\delta K_{\mathrm{s}}$.

More details about this cross-matching step, as well as the cross-matching criteria used can be found in Delmotte et al. (2001). Nearly $80 \%$ of the LMC strips have a match rate better than $90 \%$. The strips with a match rate smaller than $80 \%$ correspond to the gaps in the 2MASS data. We checked the distance distribution of the matches, by wether they were done in $J$, or $K_{\mathrm{s}}$ or both. There seems to be no relation between the magnitude criterion applied and the distance of the cross-matched source. Figure 3 shows the results of the cross-matching between DCMC and 2MASS, whatever the magnitude criterion was. The mean positional offset between matches is $0.52^{\prime \prime}$ and the modal offset is $0.25^{\prime \prime}$. Figure 4 displays the histograms of the shifts between DCMC and 2MASS in RA and Dec (in arcseconds) for the 119 strips covering the LMC. To check the results, we also compared the distribution of the close matches $\left(\leq 2^{\prime \prime}\right)$ and far matches $\left(\geq 4^{\prime \prime}\right)$ in both the $\left(J-K_{\mathrm{s}}, K_{\mathrm{s}}\right)$ colourmagnitude diagram and (RA, Dec) plane. Far matches do not show any strange physical behavior and are, as expected, distributed along lines associated with the borders of the strips suffering from field distortions, and also in the center of the Cloud where the density is higher.

\section{Comparing DENIS and 2MASS photometric systems}

We considered the mean linear relation between DCMC and 2MASS magnitudes, restricting to the range $[10,14]$ in $J$ and

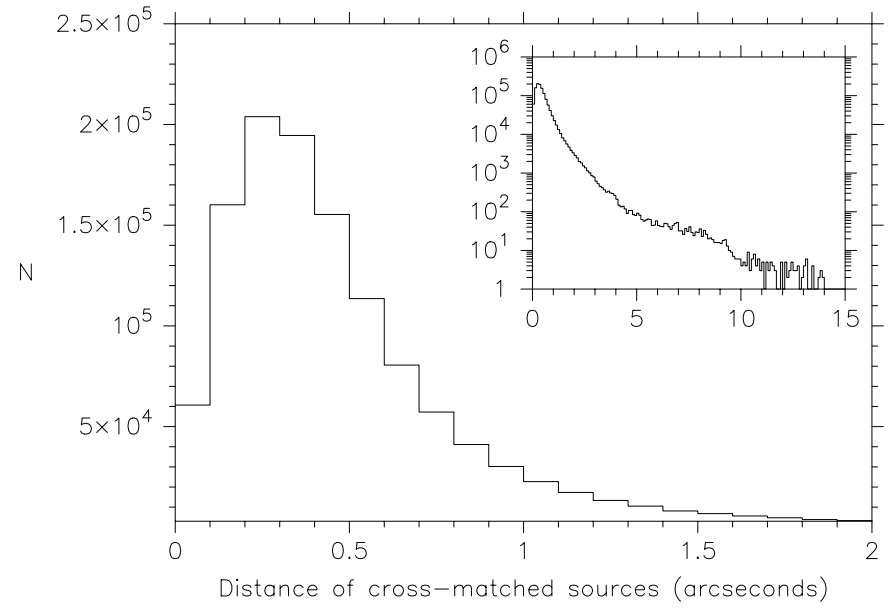

Fig. 3. Results of the cross-matching between DCMC and 2MASS. Number of objects as a function of the distance of the cross-matched point sources. The bin size is $0.1^{\prime \prime}$.

$[8,12]$ in $K_{\mathrm{s}}$, avoiding the saturated bright stars as well as the faintest ones.

We find a systematic shift of the absolute calibration between the two catalogues. For each strip, we calculated the median of $\delta J$ and $\delta K_{\mathrm{s}}$. Figure 5 shows the histograms of the shifts found for the 119 LMC strips.

The derived mean relations between the two systems are as follow:

$J_{\mathrm{DCMC}}=J_{2 \mathrm{MASS}}-(0.11 \pm 0.06)$

$K_{\text {sDCMC }}=K_{\text {s2MASS }}-(0.14 \pm 0.05)$.

These relations have been computed in the case of the DCMC catalogue and may not be valid for the whole DENIS survey. This is quite different from the relations proposed by Carpenter (2001), based on a limited preliminary sample of a few DENIS sources. Groenewegen (2000) did the comparison for a few hundred Cepheids towards the Clouds and found no significant difference between 2MASS and DENIS $J$, but found a 0.2 mag shift for the $K_{\mathrm{s}}$ band data. This larger shift compared to the one we find is probably due to the use of only variable stars (i.e. Cepheids).

\section{Second step: Adding GSC2.2 and UCAC1}

The GSC2.2 has also been divided in strips, to be crossmatched with 2MASS. These strips are no more related to the DENIS strips, but to strips with much smaller extension $\left(6^{\prime}\right)$ in order to optimize the time needed to cross-match the catalogues. We chose a searching box of $10^{\prime \prime}$. It was not possible to compare the GSC2.2 and 2MASS magnitudes in the same way as for the DCMC vs. 2MASS cross-matching (as in Sect. 3.1.3.) because there is no common magnitude between the two surveys. As a consequence, we kept only the associations to the nearest neighbour and then we cut the resulting distribution at a distance of $4^{\prime \prime}$ (Fig. 6). The mean positional offset between matches is $0.45^{\prime \prime}$ and the modal offset is $0.25^{\prime \prime}$.

At this stage, both the DCMC and the GSC2.2 are crossmatched with the 2MASS catalogue. Thus the link between the 

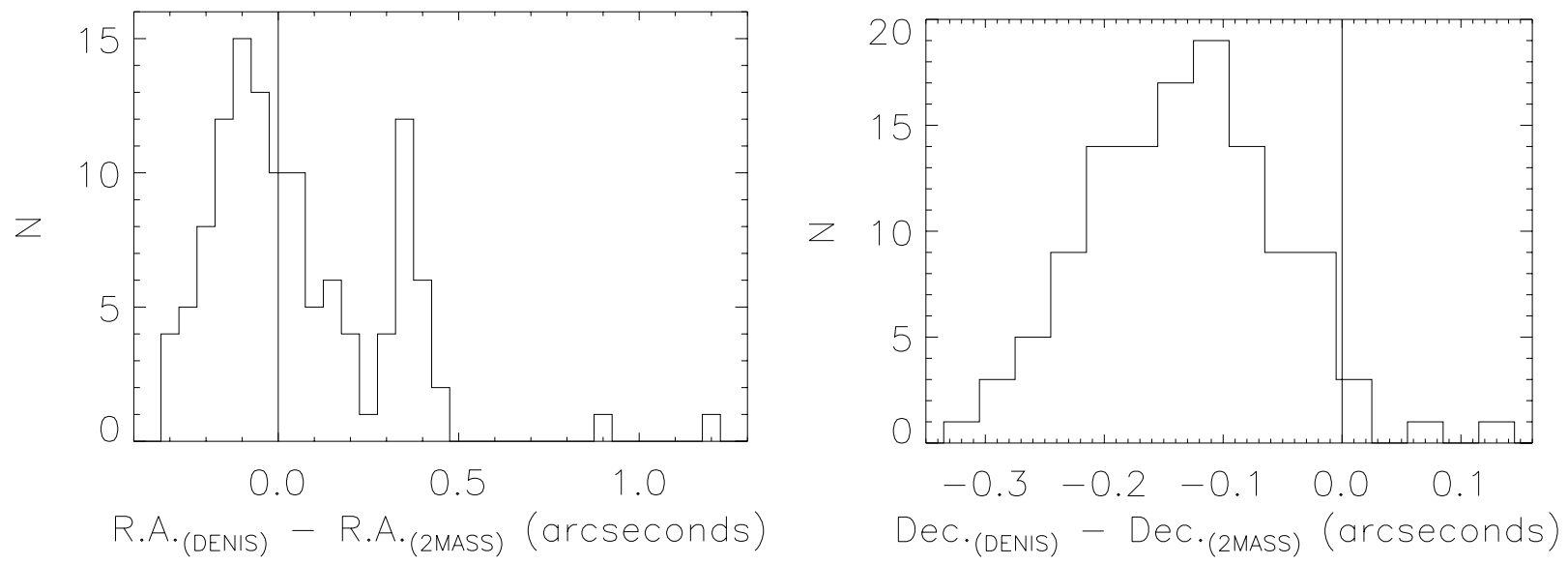

Fig. 4. Histograms of the shifts between DCMC and 2MASS RA and Dec (in arcseconds) for the 119 strips covering the LMC. The bin size is $0.05^{\prime \prime}$ for RA and $0.03^{\prime \prime}$ for Dec.
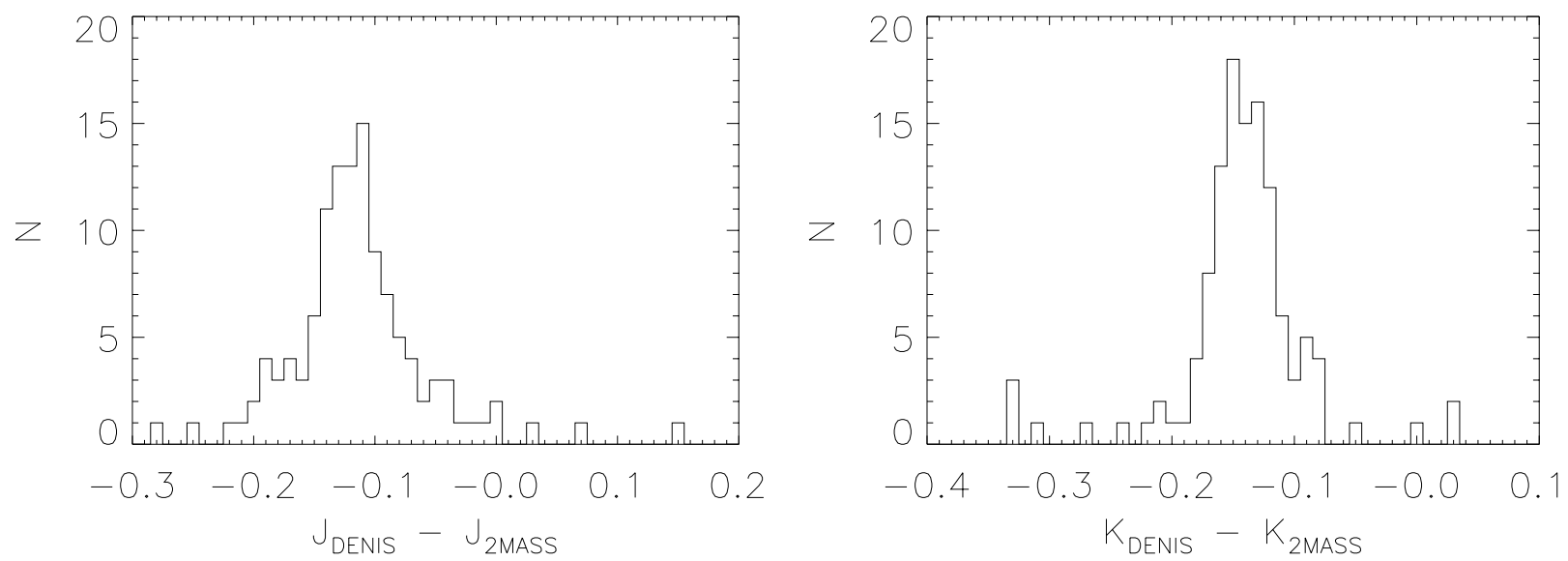

Fig. 5. Histograms of the shifts between DCMC and 2MASS $J$ and $K_{\mathrm{s}}$ magnitudes for the 119 strips covering the LMC. The bin size is $0.01^{\prime \prime}$.

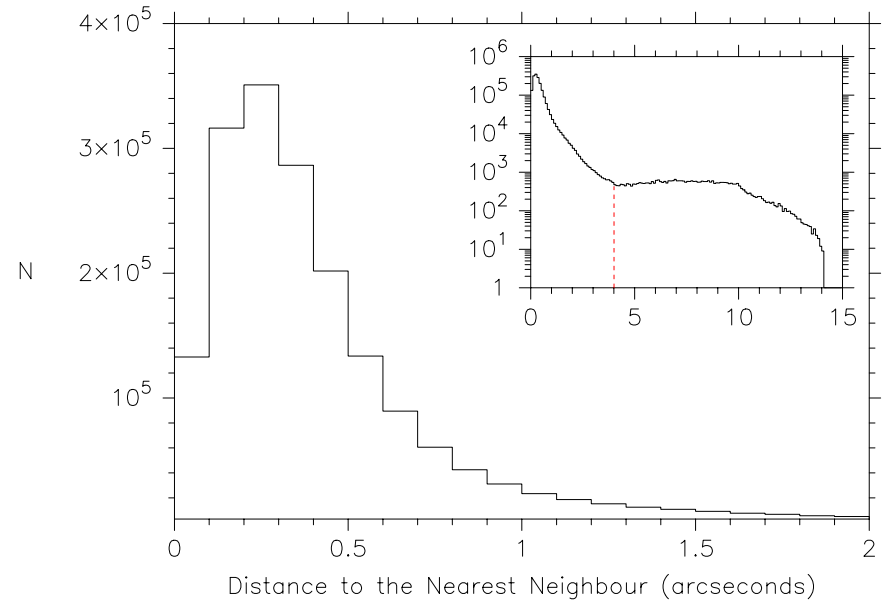

Fig. 6. Results of the cross-matching between the GSC 2.2 and the 2MASS catalogues. Number of objects as a function of the distance to the nearest neighbour. The bin size is $0.1^{\prime \prime}$.

DCMC and the GSC2.2 can be done using the 2MASS common point sources. Common entries in DCMC/2MASS and GSC2.2/2MASS have been merged. We have now six resulting files:

- sources present in DCMC, 2MASS and GSC2.2;
- sources present in DCMC and 2MASS only;

- sources present in GSC2.2 and 2MASS only;

- sources in 2MASS only;

- sources in the DCMC only;

- sources in the GSC2.2 only.

We run another cross-matching process on the last two files to find sources which are present in both DCMC and GSC2.2. For small positional differences the associations are likely true whereas at larger distances they are generally random associations, see the distribution of the sources as a function of the distance from the nearest neighbour (Fig. 7). The mean positional offset between matches is $0.61^{\prime \prime}$ and the modal offset is $0.35^{\prime \prime}$.

However, because about $1 \%$ of the DCMC sources suffer from astrometric problems, we cannot exclude that there are some true associations for sources at larger distances. Unfortunately there is no magnitude in common between the DCMC and the GSC2.2 catalogue and we cannot adopt the same strategy as in Sect. 3.1.3. We decided to keep only the associations with distances smaller than $2^{\prime \prime}$, to avoid polluting the MC2 with too many false associations. On the other hand, we are losing a few associations, located in the poorly calibrated regions of the DCMC (see the strip like features on the left plot of Fig. 8). A comparison of the slopes of the 


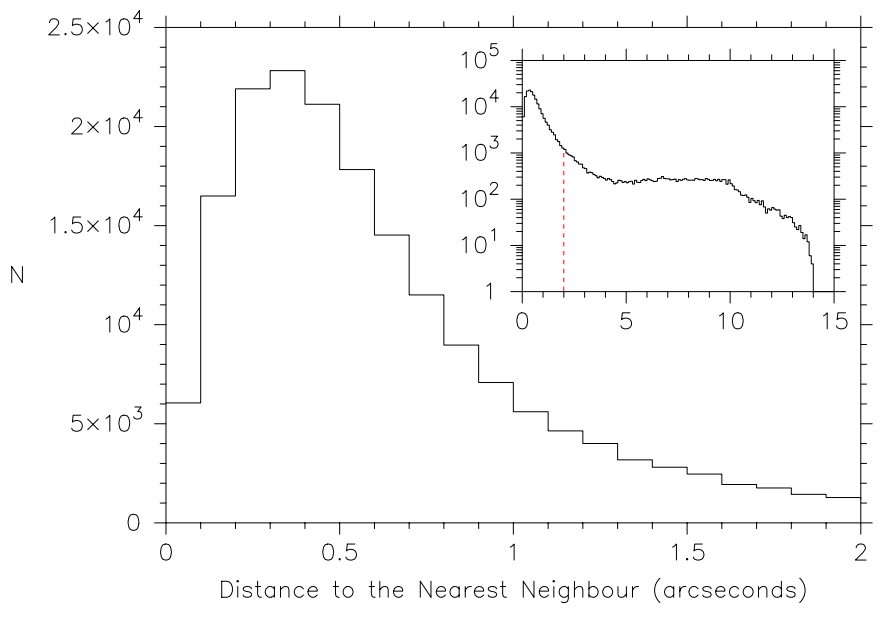

Fig. 7. Results of the cross-matching between the DCMC and the GSC2.2 sources not present in 2MASS. Number of objects as a function of the distance to the nearest neighbour. The bin size is $0.1^{\prime \prime}$.

histograms in Figs. 6 and 7 does not seem to indicate any excess of false matches for distances between $2 "$ and $4 "$. Sources belonging to both DCMC and GSC2.2, but not 2MASS, are shown on the middle plot of Fig. 8. They correspond mainly to sources falling in the yet empty gaps of the 2MASS data. The 2MASS scanning strategy covered the sky with tiles 6 degrees long in Dec and 8.5' wide in RA. These patterns remain visible on the right plot of Fig. 8, showing the spatial distribution of the MC2 sources belonging to the 2MASS catalogue only, thus denoting different sensitivity limits.

Table 2 summarizes the results obtained so far during the process to build up the MC2, which contains more than 6 million sources for the LMC. The optical/IR database contains 1968360 sources: IR from 2MASS (629212), DCMC (177414), or both (1 161734). Among the remaining sources, 4 million of them are only detected in the GSC2.2. It is of great astronomical interest to get as many wavelengths as possible for each star, but this should not lead to disregard sources detected only with one survey and not with the other ones. Keeping non-associations in the MC2 helps keeping track of the internal discrepancies and different sensitivity limits of each catalogue.

The procedure to add the UCAC1 is quite different. We cross-matched the UCAC1, without splitting it in strips, with the MC2 at its present stage. This is possible because the

Table 2. Distribution of the MC2 sources, prior the inclusion of UCAC1.

\begin{tabular}{c|c|c|r}
\hline \hline 2MASS & DCMC & GSC2.2 & Number of sources \\
\hline$\checkmark$ & $\checkmark$ & $\checkmark$ & 1161734 \\
$\checkmark$ & $\checkmark$ & & 54584 \\
$\checkmark$ & & $\checkmark$ & 629212 \\
$\checkmark$ & & & 151215 \\
& $\checkmark$ & & 65709 \\
& & $\checkmark$ & 4064181 \\
& $\checkmark$ & $\checkmark$ & 177414 \\
\hline \multicolumn{3}{|c|}{ Total }
\end{tabular}

UCAC1 is a small catalogue and our program is fast enough to process it in one run. Another advantage is that the UCAC1 is automatically cross-matched with the DCMC-only sources and the GSC2.2-only sources.

Figure 9 shows the same as Figs. 6 and 7 for UCAC1 and MC2 sources. We decided to keep all these associations, even the ones for sources at distances larger than $1^{\prime \prime}$, because these sources also display a larger proper motion compared to the average source in the catalogue (Fig. 10). This might as well be the cause of the large distance derived during the association process. The mean positional offset between matches is $0.17^{\prime \prime}$ and the modal offset is $0.15^{\prime \prime}$. About 42 UCAC1 sources do not have a MC2 counterpart, which means that $99.9 \%$ of the UCAC1 catalogue is linked to the MC2 and $4.2 \%$ of the MC2 has a UCAC1 counterpart.

\section{Results: Multispectral views of the LMC}

This paper mainly deals with the techniques that went into the construction of the MC2. It shows how essential a tool the cross-matching of large surveys is, to derive results on their internal accuracy. The broad range of magnitudes covered by the MC2, as well as the large number of sources involved, allow a multi-wavelength and statistical study of the stellar populations of the Clouds. We present a few results concerning their location in several colour-magnitude and colour-colour diagrams, in order to demonstrate the usefulness of such an optical/infrared catalogue and its relevance in the framework of the Virtual Observatory. Note that observations of crossmatched sources were not simultaneously performed so those following diagrams should be considered as indicative because the colours might not represent correctly variable sources.

\subsection{The $\left(K_{\mathrm{s}}, J-K_{\mathrm{s}}\right)$ colour-magnitude diagram}

Figure 11a shows the $\left(K_{\mathrm{s}}, J-K_{\mathrm{s}}\right)$ diagram for all the 2MASS point sources. The total number of sources, nearly two millions, was so large that we chose to plot them as isodensity curves, so as to emphasize different loci of stars. The same technique has been adopted for most of the following diagrams. Unfortunately, this process tends to hide regions with low density of stars. Sources in regions with density lower than the value of the lowest contour level have been plotted as single dots.

The 2MASS colour-magnitude diagram (CMD) has been described in details by Nikolaev \& Weinberg (2000), and it will be taken as a reference for the further discussion on the stellar populations obtained from the MC2. Figure $11 \mathrm{~b}$ is a similar CMD, but for all the DCMC point sources. Figure 11c shows the CMD of the point sources that do have a counterpart in all three catalogues: DCMC, 2MASS and GSC2.2. Figure 11d shows the CMD of all the point sources detected in both DCMC and 2MASS, but not GSC2.2. For Figs. 11c and d the $J$ and $K_{\mathrm{s}}$ magnitudes are from 2MASS, including DCMC sources detected only in $I$ and $J$.

All the 2MASS sources that do not have any counterpart have been plotted on the CMD of Fig. 11e. This feature is a mix of Asymptotic Giant Branch (AGB) and Red Giant Branch 

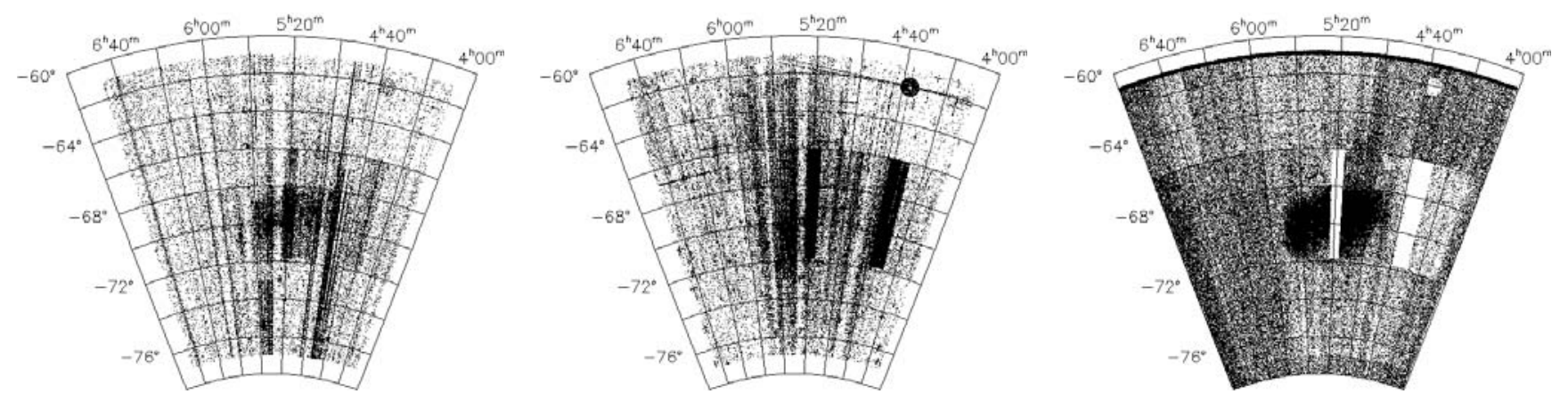

Fig. 8. Spatial distribution of some cross-matched sources. Left panel: sources of the MC2 belonging to the DCMC catalogue only. The DENIS strip structure remains visible, because some of them suffer from field distortion all along the borders. Middle panel: sources of the MC2 present only in the DCMC and the GSC2.2 catalogues. Most of them fill the gaps of the 2MASS catalogue. Right panel: sources of the MC2 belonging to the 2MASS catalogue only.

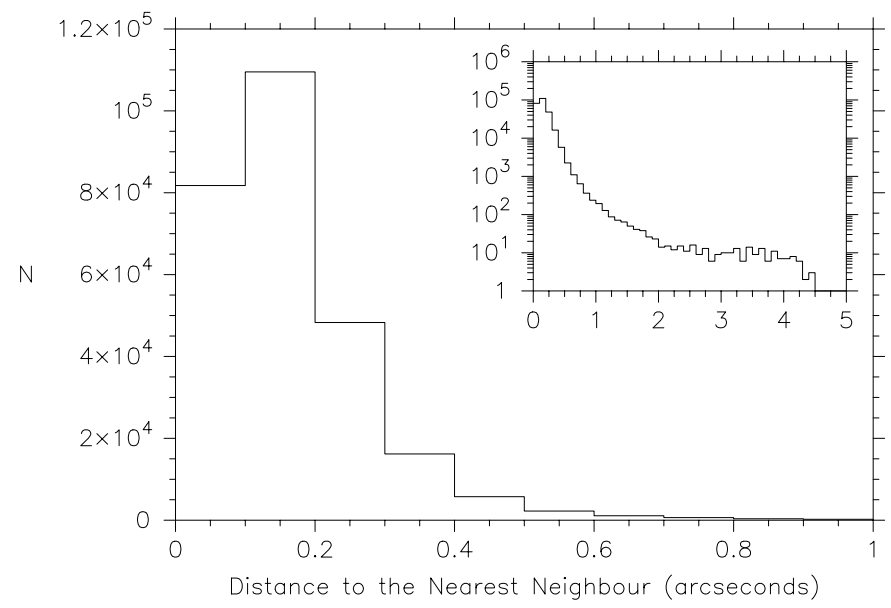

Fig. 9. Results of the cross-matching between the UCAC1 and the MC2 catalogues. Histogram of distances to the nearest neighbour. The bin size is $0.1^{\prime \prime}$.

(RGB) stars. The position of the AGB bump, located at the bottom of the AGB phase (see Gallart 1998 and references therein), was found by Nikolaev \& Weinberg (2000) in the deep 2MASS observations at $K_{\mathrm{s}}=15.8$ and $\left(J-K_{\mathrm{s}}\right)=0.7$. The AGB bump stellar population has been well identified by Alcock et al. (2000) thanks to their 9 million LMC stars resulting from the MACHO project. Note that Beaulieu \& Sackett (1998) call them the Supraclump. The sensitivity limit is too low here to detect it, as for the red clump, which is located more than one magnitude below the AGB bump $\left(K_{\mathrm{s}} \sim 17\right.$ and $\left(J-K_{\mathrm{s}}\right) \sim 0.65$, Nikolaev \& Weinberg 2000).

Figure 11f refers to sources detected in both 2MASS and UCAC1. It shows mainly a concentration of stars around $\left(J_{-}\right.$ $\left.K_{\mathrm{S}}\right)=0.5$ and $K_{\mathrm{s}}=14$, which falls into region D of Nikolaev $\&$ Weinberg (2000). Note that Nikolaev \& Weinberg (2000) associate the blue half part of region D with G-M dwarfs of the Galaxy. Ruphy et al. (1997) investigated the separation in ( $J-$ $K_{\mathrm{s}}$ ) between dwarfs and giants, with the help of early DENIS data, in the direction of the anticenter. They find that roughly for $\left(J-K_{\mathrm{s}}\right) \leq 0.6$ there could not be any giants. However, $\mathrm{K}$ and $\mathrm{M}$ dwarfs may be present for redder colours, together with the giants. RGB stars at the tip of the RGB and AGB stars both

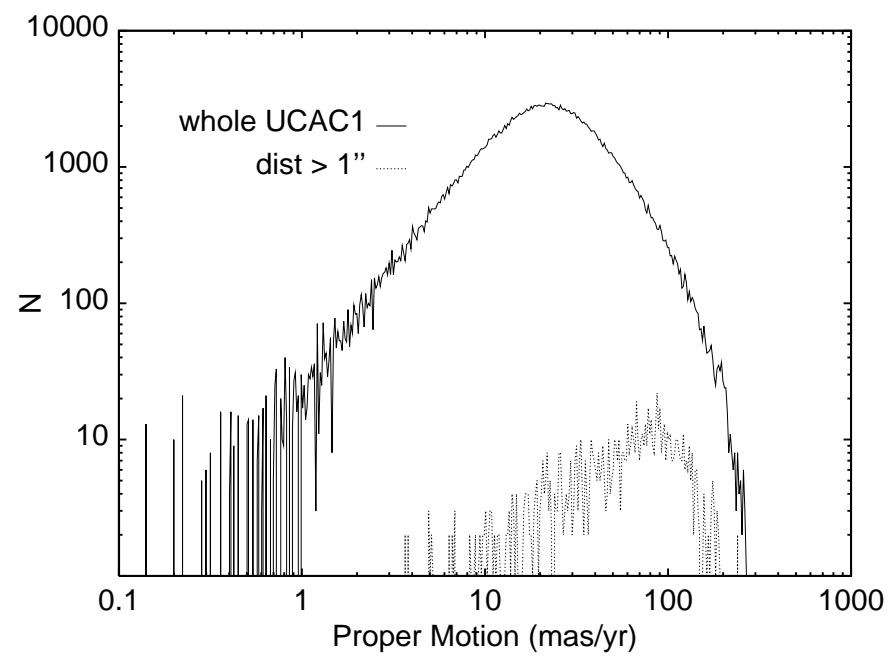

Fig. 10. Results of the cross-matching between the UCAC1 and the MC2 catalogues. Histogram of the proper motions. Both axes have logarithmic scale and the bin size is also logarithmic. The dashed line refers to the cross-matched sources with distances larger than $1^{\prime \prime}$. Their distribution is in the region of sources with large proper motions, when compared to the whole UCAC1 distribution (solid line).

O-rich and C-rich can be distinguished at $\left(J-K_{\mathrm{s}}\right) \geq 1.0$ (Cioni et al. 2000c).

\subsection{The $\left(\mathrm{J}-\mathrm{H}, \mathrm{H}-\mathrm{K}_{\mathrm{s}}\right)$ colour-colour diagram}

The $\left(J-H, H-K_{\mathrm{s}}\right)$ diagram may be used to discriminate between dwarf and giant stars, at least to find the bifurcation between M dwarfs and M giants (Bessel \& Brett 1988). Dwarfs are on the bluest peak, whereas giants are on the reddest one. This diagram contains only point sources with photometric errors on $J, H$ and $K_{\mathrm{s}}$ smaller than $0.06 \mathrm{mag}$. The $(J-H, H-$ $K_{\mathrm{s}}$ ) diagram is also suitable to isolate reddened stars. Nikolaev $\&$ Weinberg (2000) provide such a diagram and label the areas of unusual objects such as Wolf-Rayet stars, protostars, AGB C-rich stars and Be stars. Frogel et al. (1990) surveyed several Magellanic Cloud clusters and overplotted on their resulting $\left(J-H, H-K_{\mathrm{s}}\right)$ diagram the mean relations for globular cluster and field giants. The distribution of their cluster 


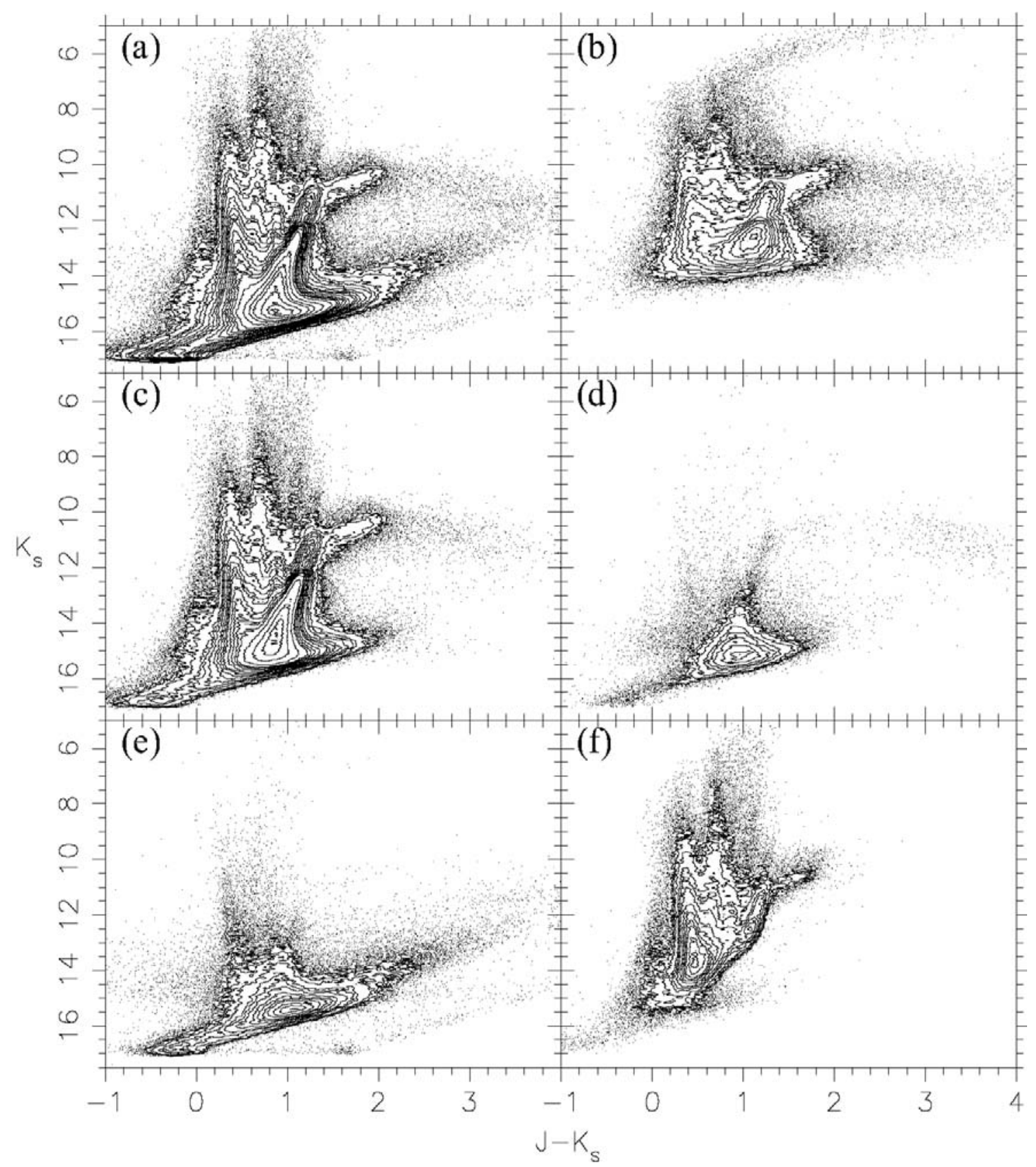

Fig. 11. a) $\left(K_{\mathrm{s}}, J-K_{\mathrm{s}}\right) 2 \mathrm{MASS}$ CMD: 1996448 entries. b) $\left(K_{\mathrm{s}}, J-K_{\mathrm{s}}\right)$ DENIS CMD: 298928 entries. c) Point sources present in 2MASS, DCMC and GSC2.2: 1161701 entries. The $J$ and $K_{\mathrm{s}}$ bands are from 2MASS. d) Point sources detected in 2MASS, DCMC but not GSC2.2: 54579 entries. The $J$ and $K_{\mathrm{s}}$ bands are from 2MASS. e) Point sources detected in 2MASS only: 151120 entries. f) Point sources detected in both the IR catalogues and UCAC1: 192848 entries. The $J$ and $K_{\mathrm{s}}$ bands are from 2MASS.

$\mathrm{M}$ giants is spread between these two lines, which they relate to a metallicity effect. This could provide an explanation to the slight shift between the giant peak in Fig. 12 and the giant track from Wainscoat et al. (1992) overplotted on it. Finlator et al. (2000) cross-matched 2MASS with SDSS, thus selecting stars on their optical colours and then tracing them in infrared diagrams. They found out that the dwarf peak in the $\left(J-H, H-K_{\mathrm{s}}\right)$ diagram is associated with stars earlier than G5, whereas the giant peak is associated with stars later than K5. This is a typical example illustrating the usefulness of the combination of optical with infrared colours, in order to separate stars according to their spectral type.

\subsection{IR/optical colour-colour diagrams}

Combining IR with optical wavelength, as shown in Fig. 13, enables us to discriminate between dwarf and giant stars.

The two peaks show the combined effect of the fact that the contribution of the Galactic foreground stars are most likely due to the bluest dwarfs than to the reddest ones, and that the limiting magnitude of the surveys excludes most LMC dwarfs. Otherwise, if all the populations of stars were present, the two peaks would be merged. The separation between these two main clusters of stars is much better than in the $(J-$ $H, H-K_{\mathrm{s}}$ ) diagram. Note that we plotted only sources with photometric errors on $I, J$ and $K_{\mathrm{s}}$ smaller than $0.06 \mathrm{mag}$. 


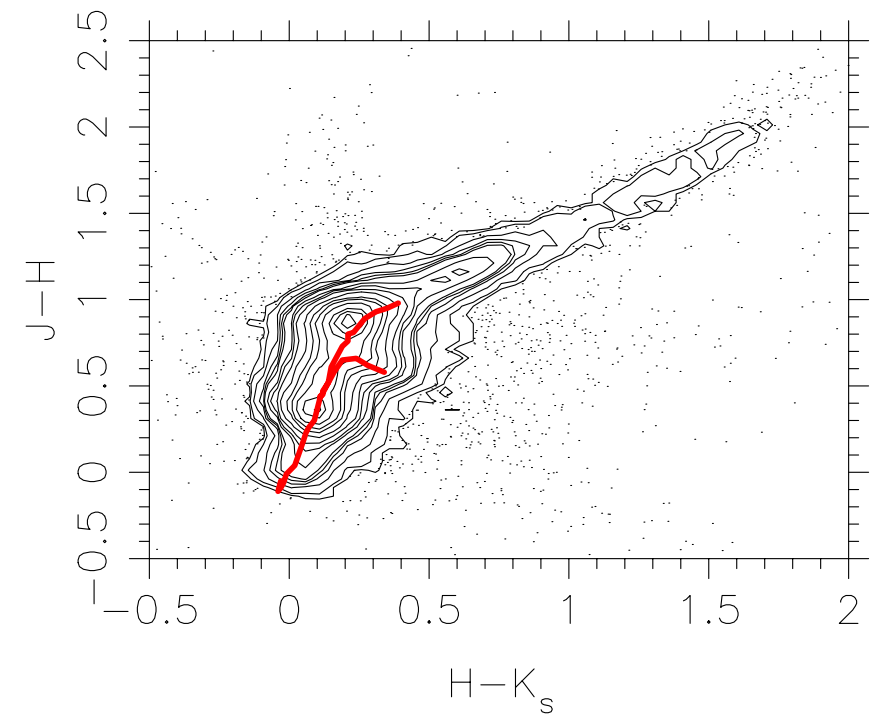

Fig. 12. Point sources detected in 2 MASS, whatever the detection in the other catalogues is: 423445 entries (photometric errors smaller than $0.06 \mathrm{mag}$ ). The colour/colour dwarf and giant tracks are computed using Table 2 from Wainscoat et al. (1992).

Two vertical sequences appear at $\left(J-K_{\mathrm{s}}\right)=0.9$ (dwarfs) and $\left(J-K_{\mathrm{s}}\right)=1.25$ (giants). We identify the bluest vertical sequence with late $\mathrm{M}$ dwarfs, as suggested by the tracks superimposed on the $\left(I-J, J-K_{\mathrm{s}}\right)$ and $\left(V-J, J-K_{\mathrm{s}}\right)$ diagrams. Note that the colour/colour giant track of both Wainscoat et al. (1992) and Bessel \& Brett (1988) do not exactly match the MC2 data. The shift is roughly 0.1 magnitude in $\left(J-K_{\mathrm{S}}\right)$, which could be a photometric calibration problem. However it does not affect the track for the dwarfs which are mostly galactic foreground stars. As a consequence, since it affects only the track for the giants, it might be due to metallicity or extinction effect. The search for late $\mathrm{M}, \mathrm{L}$ and $\mathrm{T}$ dwarfs has been successful since the beginning of near-infrared sky surveys. But as pointed out by Leggett et al. (2002), infrared photometry alone does not allow to clearly discriminate between the different spectral types. It is much easier to identify them on the basis of their optical/infrared colour index (see also Kirkpatrick et al. 1999), because they are so faint in the optical, and comparatively much brighter in the IR. These stars should disentangle themselves from the usual stars, and Reid et al. (2001) provide the location of some of these stars in the $\left(I-J, J-K_{\mathrm{s}}\right) \mathrm{CMD}$ (and also $(J-H$, $\left.\left.H-K_{\mathrm{s}}\right)\right)$. Smart et al. (2001) have stressed out the value of the GSC2 in the search for ultracool stars.

Some other well defined features (such as the $\mathrm{M}$ giant $\mathrm{O}$-rich star and the $\mathrm{C}$-star sequences) appear on each panel of Fig. 13, especially on the $\left(V-J, J-K_{\mathrm{s}}\right)$ diagram, where the spectral range between the optical and infrared magnitudes is much broader.

\subsection{The $\left(I, V-K_{s}\right)$ colour-magnitude diagram}

We computed several CMDs using a combination of three different wavelengths, both IR and optical, out of the different catalogues. The best features are obtained with the $V_{\mathrm{gsc} 2}, I$, and $K_{\mathrm{s}}$ bands (Fig. 14).
The red supergiants (SGs) are located in the tight upward sequence at $I \sim 14$ and $\left(V-K_{\mathrm{s}}\right) \sim 3$, while the blue SGs have $\left(V-K_{\mathrm{s}}\right) \leq 1$. This is consistent with the evolutionary tracks from Girardi et al. (2000). We looked at the distribution of the stars with $\left(V-K_{\mathrm{s}}\right) \leq 1$ in various diagrams. The results are summarized in Fig. 15. They belong to the central parts of the LMC, and their spatial distribution is clumpy (Fig. 15d), quite similar to what Martin et al. (1976) had found with their merging of several catalogues containing SG stars. These sources are linked to the supergiant shells of the LMC (Meaburn 1980), which are probably produced by the effect of stellar winds and/or supernovae. These stars should help us constraining the recent star formation history of the LMC (Grebel \& Brandner 1998; Dolphin \& Hunter 1998). Some of them fall into region A of Nikolaev \& Weinberg (2000) (Fig. 15a): blue SGs, O dwarfs. Since they are very blue stars, their $\left(V-K_{\mathrm{s}}\right)$ colour distinguish them from the bulk of stars on the $\left(I, V-K_{\mathrm{s}}\right)$ diagram (Fig. 14). They match the overdensity of stars at $(I-J)=-0.25$ and $(J-$ $\left.K_{\mathrm{s}}\right)=0$ and extend towards redder colours (Fig. 15b). They are also recognizable on the $\left(J-H, H-K_{\mathrm{s}}\right)$ diagram at $(0,0)$, at the bottom of the sequence of dwarfs (Fig. 15c). These young stars are much more easy to trace in the IR/optical colour-colour diagrams and CMDs than in the $\left(K_{\mathrm{s}}, J-K_{\mathrm{s}}\right)$ CMD.

\section{Conclusion}

The Master Catalogue of stars towards the Magellanic Clouds (MC2) is now available on the web at $\mathrm{CDS}^{5}$. It is a compilation of cross-identified surveys, from optical to IR. The MC2 roughly covers the following area: $4^{\mathrm{h}}$ to $7^{\mathrm{h}}$ in Right Ascension, and $-61^{\circ}$ to $-78^{\circ}$ in Declination, with slight variations according to the catalogue considered.

We are currently working on the cross-identification of analogous catalogues in the direction of the Small Magellanic Cloud and we plan to add catalogues and tables at other wavelengths: ROSAT, IRAS, and many more specific catalogues, as well the variability informations coming either from MACHO, EROS, OGLE to the second version of the catalogue.

A typical query of the MC2 returns several lines of data. An example is given in Table 3. Each line of data contains the name of the source for all the original catalogues, followed by the magnitudes and the proper motion when the UCAC1 is present. For each catalogue, the distance of the cross-identification is given, except for 2MASS which is taken as reference. The distance associated to a DCMC source is the distance to the 2MASS counterpart. The distance associated to a GSC2.2 source is the distance to the 2MASS counterpart, or the DCMC counterpart when there is no 2MASS counterpart. The distance associated to a UCAC1 source is the distance to the MC2 counterpart (2MASS, DCMC or GSC2.2, depending on the detection bands). At the beginning of each line, RA and Dec are given. The choice of the coordinates has been as follows: when possible, we kept the 2MASS coordinates as the reference, otherwise we took the GSC2.2, then the UCAC1 and finally the DCMC ones.

\footnotetext{
${ }^{5}$ http://vizier.u-strasbg.fr/MC2/
} 

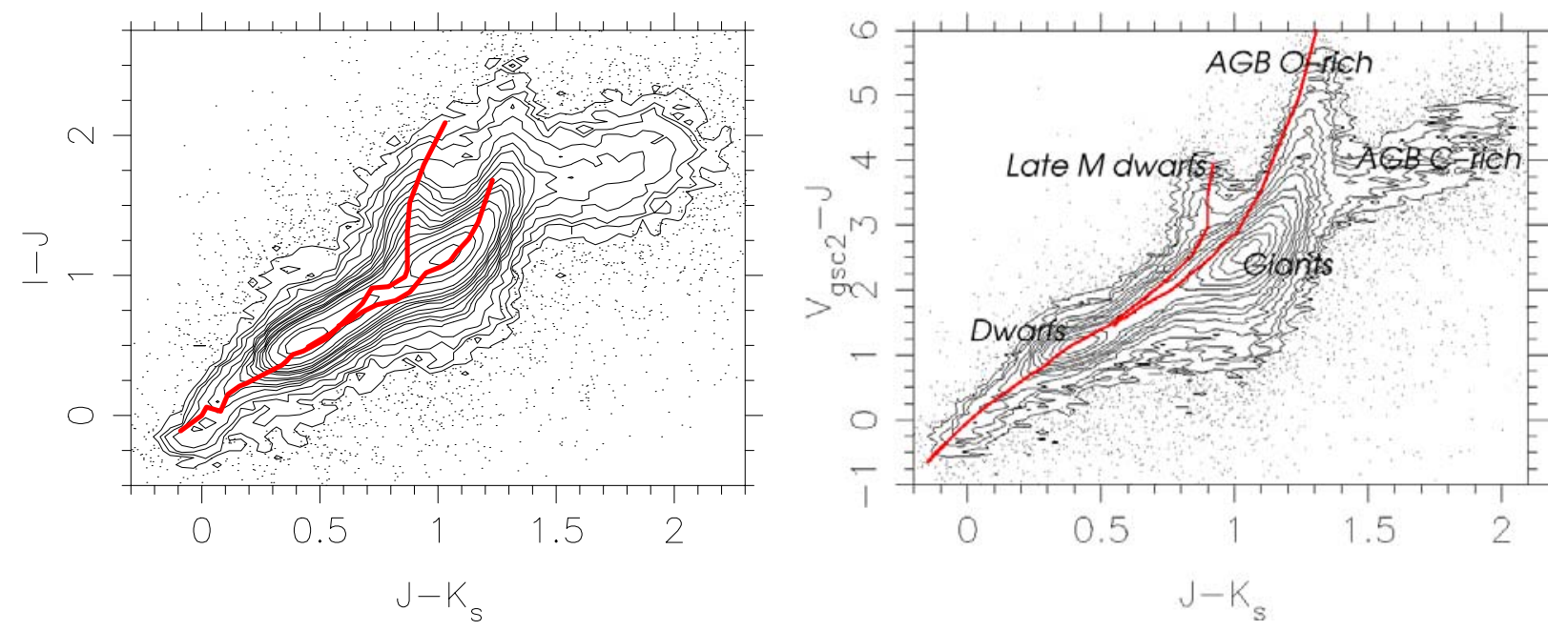

Fig. 13. Panel a) contains sources detected by both DCMC and 2MASS: 372354 entries. The $I$ band is from DENIS, whereas the $J$ and $K_{\mathrm{s}}$ bands are from 2MASS. Dwarf and giant tracks superimposed are from Bessel \& Brett (1988). Panel b) contains sources detected by both GSC2.2 and 2MASS: 147564 entries. Dwarf and giant tracks are computed using Table 2 from Wainscoat et al. (1992). The sources involved in those diagrams have photometric errors smaller than 0.06 mag.

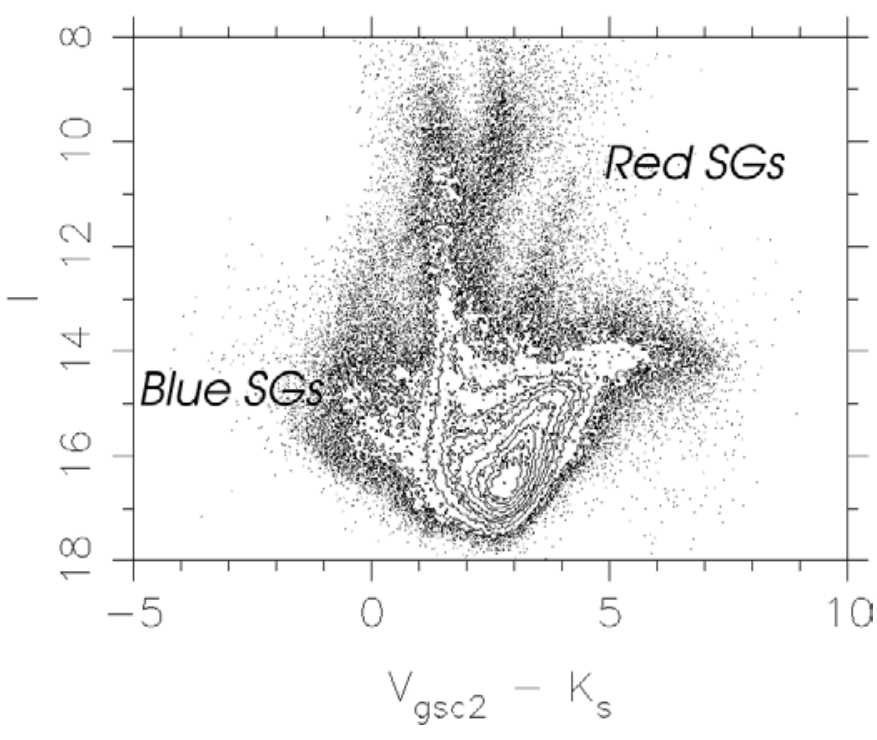

Fig. 14. CMD resulting from the cross-matching between the DCMC $(I), 2 \mathrm{MASS}\left(K_{\mathrm{s}}\right)$ and GSC2.2 $\left(V_{\mathrm{gsc} 2}\right)$ catalogues. This plot contains 393179 entries.

We decided to keep the distances of the crossidentifications in the MC2 to give the user the opportunity to judge the reliability of each cross-identification. We have also shown that for some DCMC point sources, this distance was not reliable. But since links for each source allow to access the complete data from the original catalogues through the VizieR search engine (Ochsenbein et al. 2000), it is always possible to retrieve the strip number of the DCMC source and then go to the MC2 web site to find the shifts associated to this strip. Those links are also very valuable in order to retrieve observational data such as image or scan number, flags or whatever parameter the user would like to know from the original catalogues.
This reference catalogue is made available as a support for a number of studies concerning, e.g. the stellar populations in the Magellanic Clouds, the structure of the Clouds, or certain classes of objects (Cepheids, AGB stars, etc.). Recent articles, such as those by Zaritsky et al. (2002), van der Marel (2001), Nikolaev \& Weinberg (2001) and Cioni et al. (2000b) have demonstrated the power of optical and near-infrared surveys to improve our understanding on these neighbouring galaxies.

Acknowledgements. We thank an anonymous referee for very constructive suggestions which helped to improve the present paper. We would like to thank François Ochsenbein for his help with the catalogues. This research has made use of the SIMBAD astronomical database, the VizieR catalogue service, and the ALADIN interactive sky atlas, all operated at CDS, Strasbourg, France. This work has been partly supported by the ASTROVIRTEL Project which is run by the ESO/ST-ECF Archive and funded by the European Commission under contract HPRI-CT-1999-00081. This publication makes use of data products from the Two Micron All Sky Survey, which is a joint project of the University of Massachusetts and the Infrared Processing and Analysis Center/California Institute of Technology, funded by the National Aeronautics and Space Administration and the National Science Foundation, and from DENIS, which is the result of a joint effort involving human and financial contributions of several Institutes mostly located in Europe. It has been supported financially mainly by the French Institut National des Sciences de l'Univers, CNRS, and French Education Ministry, the European Southern Observatory, the State of Baden-Württemberg, and the European Commission under a network of the Human Capital and Mobility program. The Guide Star Catalogue-II is a joint project of the Space Telescope Science Institute and the Osservatorio Astronomico di Torino. Space Telescope Science Institute is operated by the Association of Universities for Research in Astronomy, for the National Aeronautics and Space Administration under contract NAS5-26555. The participation of the Osservatorio Astronomico di Torino is supported by the Italian Council for Research in Astronomy. Additional support is provided by European Southern Observatory, Space Telescope European Coordinating Facility, the International GEMINI project and the European Space Agency Astrophysics Division. 

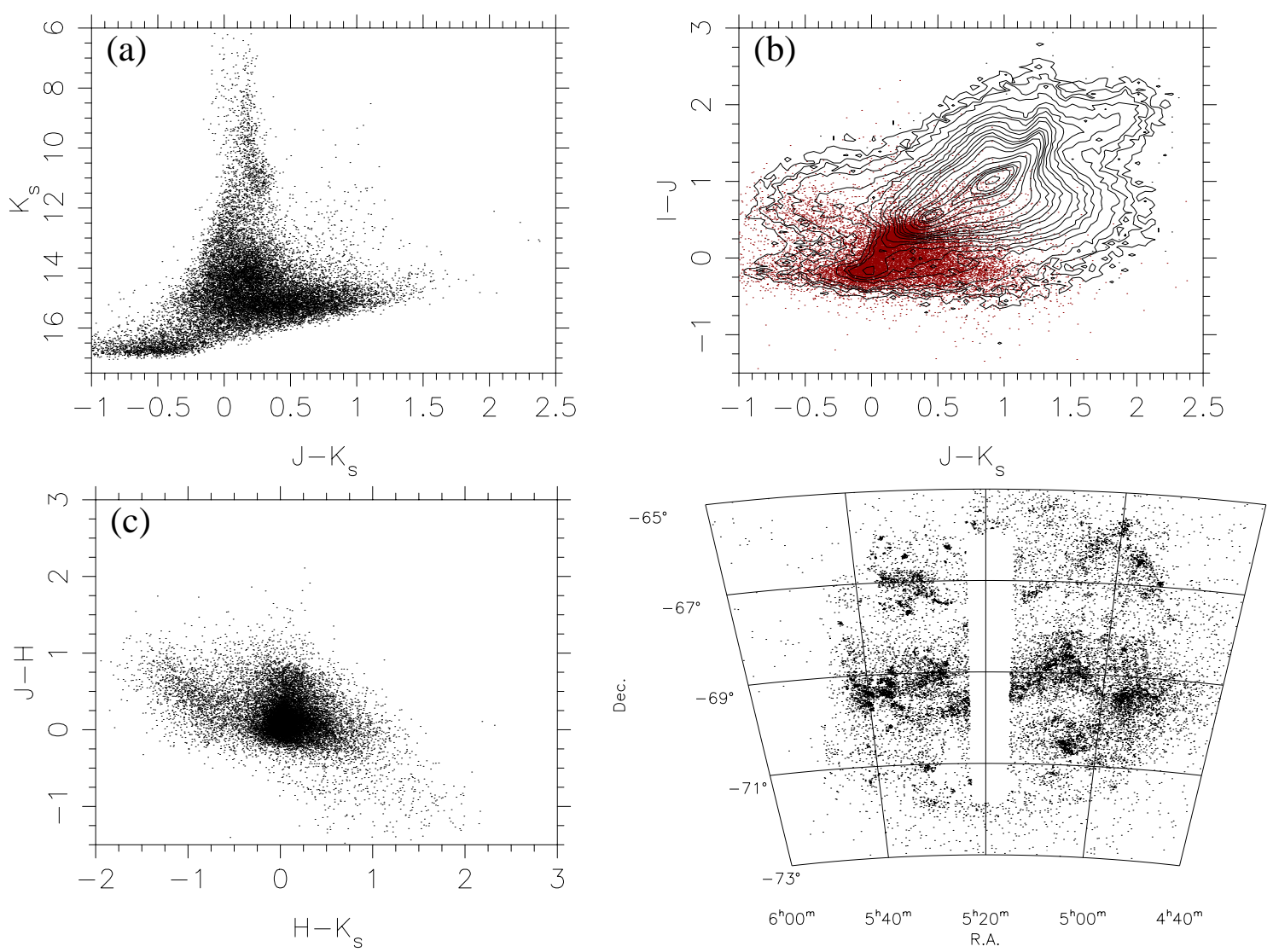

Fig. 15. CMD, colour-colour diagrams and spatial distribution of the blue stars selected with $\left(V-K_{\mathrm{s}}\right) \leq 1$ in the $\left(I, V-K_{\mathrm{s}}\right)$ diagram. There are 19646 sources on each panel. The $I$ band is from DENIS and the $J, H$ and $K_{\mathrm{s}}$ are from 2MASS. The contour levels have been added so as to show the locus of different types of objects.

Table 3. Subsample of the MC2. Each line of data corresponds to one point source.

\begin{tabular}{ccccccccc}
\hline RA & Dec & 2MASS & $J$ & $\mathrm{~d} J$ & $H$ & $\mathrm{~d} H$ & $K_{\mathrm{s}}$ & $\mathrm{d} K_{\mathrm{s}}$ \\
\hline 93.769831 & -75.633698 & $0615047-753801$ & 12.380 & 0.022 & 12.177 & 0.024 & 12.076 & 0.029 \\
93.760565 & -75.632538 & $0615025-753757$ & 16.072 & 0.089 & 15.975 & 0.178 & 15.273 & null \\
93.769231 & -75.629761 & $0615046-753747$ & 16.413 & 0.123 & 16.141 & 0.211 & 15.611 & 0.261 \\
93.756995 & -75.621536 & $0615016-753717$ & 16.192 & 0.098 & 15.352 & 0.106 & 15.443 & 0.215 \\
\hline DCMC & $I$ & $\mathrm{~d} J$ & $J$ & $\mathrm{~d} J$ & $K_{\mathrm{s}}$ & $\mathrm{d} K_{\mathrm{s}}$ & dist \\
\hline $061504.60-753800.7$ & 12.999 & 0.006 & 15.788 & 0.188 & 99.000 & 99.000 & 0.853030 \\
$061502.40-753757.5$ & 16.501 & 0.062 & 15.866 & 0.230 & 99.000 & 99.000 & 0.621811 \\
.. & $\ldots$ & $\ldots$ & $\ldots$ & $\ldots$ & $\ldots$ & $\ldots$ & $\ldots$ \\
.. & $\ldots$ & $\ldots$ & $\ldots$ & $\ldots$ & $\ldots$ & $\ldots$ & $\ldots$ \\
\hline GSC2.2 & $F$ & $\mathrm{~d} F$ & $J$ & $\mathrm{~d} J$ & $V$ & $\mathrm{~d} V$ & dist \\
\hline S1102121266 & 13.24 & \pm 0.23 & 13.95 & \pm 0.17 & - & \pm & 0.192289 \\
S11021214338 & 17.40 & \pm 0.23 & 17.90 & \pm 0.18 & - & \pm & 0.203876 \\
S11021214339 & 17.44 & \pm 0.23 & 18.29 & \pm 0.18 & - & \pm & 0.554134 \\
S11021214495 & 18.30 & \pm 0.24 & - & \pm & - & \pm & 0.251949 \\
\hline UCAC1 & mag & PMra & PMdec & dist & & & \\
\hline 00697288 & 13.22 & +27.3 & -9.7 & 0.045439 & & & \\
$\ldots$ & $\ldots$ & $\ldots$ & $\ldots$ & $\ldots$ & & & \\
$\ldots$ & $\ldots$ & $\ldots$ & $\ldots$ & $\ldots$ & & & \\
\hline
\end{tabular}

\section{References}

Alcock, C., Allsman, R. A., Alves, D. R., et al. 2000, AJ, 119, 2194 (MACHO)

Beaulieu, J. P., \& Sackett, P. D. 1998, AJ, 116, 209

Bessel, M. S., \& Brett, J. M. 1988, PASP, 100, 1134
Bucciarelli, B., García Yus, J., Casalegno, R., et al. 2001, A\&A, 368, 335 (GSPC2)

Carpenter, J. 2001, AJ, 121, 2851

Cioni, M.-R., Loup, C., Habing, H. J., et al. 2000a, A\&AS, 144, 235 (DCMC) 
Cioni, M.-R., Habing, H. J., Israel, F. P., et al. 2000b, A\&A, 358, L9

Cioni, M.-R., van der Marel, R. P., Loup, C., \& Habing, H. J. 2000c, A\&A, 359, 601

Delmotte, N., Egret, D., Loup, C., \& Cioni, M.-R. 2001, SPIE Conf. Ser., 4477, 301

Epchtein, N., de Batz, B., Capoani, L., et al. 1997, The Messenger 87, 27 (DENIS)

Dolphin, A. E., \& Hunter, D. A. 1998, AJ, 116, 1275

Finlator, K., Ivezić, Ž., Fan, X., et al. 2000, AJ, 120, 2615

Frogel, J. A., Mould, J., \& Blanco, V. M. 1990, AJ, 352, 96

Gallart, C. 1998, ApJ, 495, L43

Girardi, L., Bressan, A., Bertelli, G., \& Chiosi, C. 2000, A\&AS, 141, 371

Grebel, E. K., \& Brandner, W. 1998, Proc. of the Workshop The Magellanic Clouds and Other Dwarf Galaxies of the Bonn/Bochum-Graduiertenkolleg, 151

Groenewegen, M. A. T. 2000, A\&A, 363, 901

Kirkpatrick, J. D., Reid, I. N., Liebert, J., et al. 1999, ApJ, 519, 802

Leggett, S. K., Golimowski, D. A., Fan, X., et al. 2002, ApJ, 564, 452

Martin, N., Prévot, L., Rebeirot, E., \& Rousseau, J. 1976, A\&A, 51, 31

Meaburn, N. 1980, MNRAS, 192, 365

Monet, D., Canzian, B., Dahn, C., et al. 1998, The PMM USNO A2.0 Catalog, US Naval Observatory, Flagstaff Station
Nikolaev, S., \& Weinberg, M. D. 2000, ApJ, 542, 804

Ochsenbein, F., Bauer, P., \& Marcout, J. 2000, A\&AS, 143, 23 (VizieR)

Reid, I. N., Burgasser, A. J., Cruz, K. L., Kirkpatrick, J. D., \& Gizis, J. E. 2001, AJ, 121, 1710

Ruphy, S., Epchtein, N., Cohen, M., et al. 1997, A\&A, 326, 597

Skrutskie, M. F., Schneider, S. E., Stiening, R., et al. 1997, ASSL 210, 25 (2MASS)

Smart, R. L., Carollo, D., Lattanzi, M. G., McLean, B., \& Spagna, A. 2001, The Second Guide Star Catalogue and Cool Stars in Ultracool Dwarfs: New Spectral Types L and T, ed. R. A. Hugh Jones, \& A. Iain Steele (Berlin Heidelberg: Springer), 119

Urban, S. E., Corbin, T. E., \& Wycoff, G. L. 1998, AJ, 115, 2161 (ACT)

van der Marel, R. P., \& Cioni, M.-R. L. 2001, AJ, 122, 1807

van der Marel, R. P. 2001, AJ, 122, 1827

Wainscoat, R. J., Cohen, M., Volk, K., Walker, H. J., \& Schwartz, D. E. 1992, ApJS, 83, 111

Weinberg, M. D., \& Nikolaev, S. 2001, ApJ, 548, 712

Zacharias, N., Urban, S. E., Zacharias, M. I., et al. 2000, AJ, 120, 2131 (UCAC1)

Zaritsky, D., Harris, J., Thompson, I. B., Grebel, E. K., \& Massey, P. 2002, AJ, 123, 855 